\title{
Inmunoadsorción: descripción de una nueva técnica
}

\author{
Ángeles Mayordomo Sanz, Azucena Barranco Sos, Ma Pilar Sobrado Sobrado, Carina Caamaño Lado, Alba \\ Luz Montoya Echeverry, Antonia Vives Bonjoch
}

Hospital Clinic. Barcelona

\section{Introducción:}

La inmunoadsorción es un tratamiento que permite de forma terapéutica la eliminación de un determinado componente del plasma sanguíneo, reinfundiendo el plasma al paciente evitando así la administración de componentes extraños al organismo. Para ello utilizamos el monitor Life 18tm que con las columnas TheraSorb tm -Ig Flex consigue eliminar inmunoglobulinas humanas del tipo IgG, IgA, IgE, IgM e inmunocomplejos. En nuestro servicio esta técnica la utilizamos principalmente para la preparación del trasplante renal de vivo en grupo $A B 0$ incompatible.

\section{Objetivo:}

Describir cómo se realiza la técnica de la inmunoadsorción.

\section{Material y métodos:}

Material: Theraline cassete de tubos, separador de disco, un par de columnas TheraSorb tm Ig Flex, tarjetas identificativas de las columnas y del paciente, soluciones para la preparación, lavado y regeneración de las columnas, filtros de partículas, material para la conexión del acceso vascular (FAVI o CVC).

Método: El tratamiento sigue un esquema estándar, el cual es idéntico para todos los tipos de tratamiento de TheraSorb TM. Dicho esquema sería una sucesión de ciclos, estos ciclos se inician con la extracción de sangre del paciente a través del acceso vascular, agregaríamos anticoagulante, a continuación se produce la separación del plasma en el disco separador. Este plasma iría a rellenar la primera columna donde se produce la adsorción y filtrado, y sucesivamente el plasma retorna al paciente con el resto de componentes sanguíneos. Mientras tanto la segunda columna se va regenerando y elimina los productos de desecho. Cada ciclo de regeneración consta de: vaciado de plasma a través de la columna con suero fisiológico, lavado intermedio con suero fisiológico, regeneración de la columna con glycina, neutralización con PBS y lavado final con suero fisiológico. Para prevenir la coagulación de la sangre o del plasma en el circuito extracorpóreo, utilizaremos la combinación de dos fármacos anticoagulantes (heparina sódica al $1 \%$ y el citrato). Uno de los efectos secundarios de la utilización del citrato es la disminución del calcio iónico en sangre. Para neutralizar dicha disminución administraremos una perfusión intravenosa de gluconato cálcico para mantener los valores del calcio iónico del paciente entre 1.03$1.2 \mathrm{mmol} / \mathrm{l}$. Para el control del calcio se realizarán analíticas horarias durante todo el proceso, además de la pre y post-tratamiento. La duración del tratamiento dependerá del valor inicial de las IgG del paciente (que determinará el número de ciclos) y del flujo de sangre que logremos alcanzar (máximo $120 \mathrm{ml} / \mathrm{min}$ ). Finalizado el tratamiento y el retorno sanguíneo del circuito extracorpóreo al paciente, se iniciará la regeneración y conservación de las columnas.

\section{Conclusiones:}

La realización de esta nueva técnica ha sido compleja y motivadora para enfermería, ya que supuso una innovación en el servicio de diálisis. La mayor dificultad encontrada ha sido lograr el equilibrio en- 
[ Ángeles Mayordomo Sanz, et al ]

Inmunoadsorción: descripción de una nueva técnica

tre el citrato y el gluconato cálcico para mantener valores estables del calcio iónico en sangre. Para el paciente no es una técnica tan agresiva cómo la hemodiálisis pues no disminuye la volemia, los flujos de sangre son bajos y la cantidad de sangre extracorpórea es mínima. 